\title{
Is Periodontitis a Risk Predictor with Adverse Outcomes for Cardiovascular Diseases: Treat Gums, Save Heart
}

\author{
Ashutosh Nirola ${ }^{1}$, Priyanka Batra ${ }^{2}$, Priyanka Singla ${ }^{3}$, Shivani Rathore ${ }^{4}$, \\ Anshum Gupta ${ }^{5}$ \\ ${ }^{1}$ Principal and HOD, Dept of Periodontology, LBDC, Patiala, India \\ ${ }^{2}$ Professor, Dept of Periodontology, LBDC, Patiala, India \\ ${ }^{3}$ Reader, Dept of Periodontology, LBDC, Patiala, India \\ ${ }^{4}$ Reader, Dept of Periodontology, LBDC, Patiala, India \\ ${ }^{5} \mathrm{PG}$ student, Dept of Periodontology, LBDC, Patiala, India \\ Corresponding Author: Ashutosh Nirola
}

\begin{abstract}
Different clinical researches have been carried out to find the relationship between cardiovascular diseases (CVD) and Periodontitis. Even the findings and study types of these studies haven't been inconsistent, most of them indicated that the periodontitis may be associated with an increased risk of Cardiovascular diseases. The purpose of this review article is to find the strength of the association between CVD and periodontitis. The current evidence supports the significant presence of association between CVD and periodontitis. Also it's seen that CVD patients had worse periodontal and oral hygiene status and fewer teeth than control subjects. Thus we need to conduct more high-quality and well-designed studies focusing on the specific/bidirectional relationship between CVD and periodontitis in the future.
\end{abstract}

Keywords: Periodontitis, Cardiovascular diseases, Risk factor, Mechanism and Association

\section{INTRODUCTION}

Cardiovascular diseases (CVD), including acute myocardial infarction and angina pectoris, are a major health problem in most countries. Coronary heart disease accounts for just under half of cardiovascular diseases deaths and with cerebrovascular disease and peripheral vascular disease, comprises the atherosclerotic vascular diseases (ASVD) ${ }^{1}$. Myocardial infarction (MI) is caused due to coronary artery occlusion which is result of atherosclerotic plaques and this MI event can further cause necrosis and apoptosis in myocardial cells leading to severe reduction in the number of myocardial cells, which can directly lead to heart dysfunction and ultimately failure ${ }^{2}$.
Periodontitis shares common risk factors with CVD, such as diabetes and smoking. In addition, systemic and local inflammation caused by periodontitis contributes to the risk of $\mathrm{CVD}^{3}$. Also the periodontal pathogens have shown to induce myocarditis in mice. As seen patients with a recent myocardial infarction, these patients have increased incidence of oral disease such as periapical lesions, dental caries, periodontitis and pericoronitis. Poor oral health was more frequently found in patients with recent strokes than in stroke-free individuals. Similarly those with periodontitis were at a greater risk of having a cardiovascular event than those without periodontitis. Studies suggest an association exists between ASVD and periodontitis 

gums, save heart.

however the association appears relatively modest with an increased risk of approximately $1.14-2.2$ times to fold ${ }^{1}$.

\section{Interrelationship between Periodontal Inflammation and Cardiovascular diseases}

Periodontal infections being associated with increased levels of associating inflammatory factors such as CRP and fibrinogen might be associated with increased risk for atherosclerotic processes such as coronary artery disease and strokes. Atherosclerosis is a disease of large and medium sized muscular arteries which is characterized by endothelial dysfunction, vascular inflammation and the buildup of lipids, cholesterol, calcium and cellular debris within the intima of the vessel wall ${ }^{4}$. This buildup results in plaque formation, vascular remodeling, acute \& chronic luminal obstruction, abnormalities of blood flow and diminished oxygen supply to target organs ${ }^{4}$.

Coronary artery disease also leads to increase of both $\mathrm{C}$ Reactive Protein (CRP) levels and leukocytosis and leukocytes take part in atherosclerosis through their participation in the inflammatory process; by causing endothelial dysfunction, microcirculation disorders, have proteolytic, oxidative, and procoagulant effect. Studies demonstrated highly statistically significant associations between increased white blood cell counts and increased levels of acute phase proteins such as fibrinogen and CRP with a subsequent risk for cardiovascular disease. Several studies have shown that increased CRP levels as valuable prognostic indicators of the risk for cardiovascular events ${ }^{5}$.

Periodontal diseases are significant predictor of subsequent CHD disease. There was a 70\% increase in the risk for hospitalization or death from a CHD-related event in individuals younger than 50 years who had periodontitis compared with similarly aged people without periodontitis. Investigators found a dose relation between various levels of bone loss $(20 \%, 40 \%, 60 \%$ and $80 \%$ ) and cumulative incidence of angina and myocardial infarction. Specific pathogenic bacteria found in periodontal disease have been proved to be associated with cardiovascular diseases ${ }^{5}$.

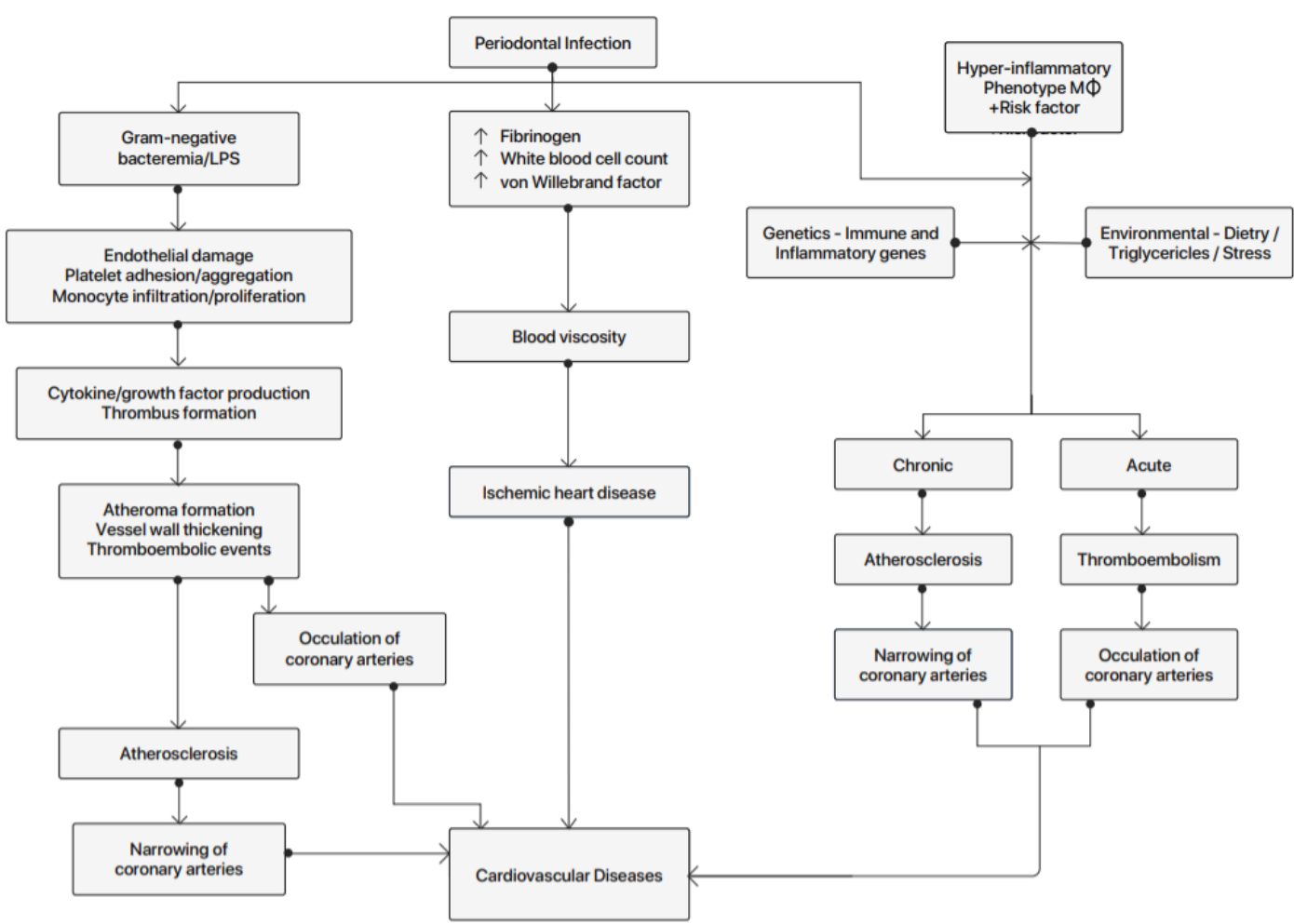

Fig 1: Interrelationship between Periodontal Inflammation and Cardiovascular Disease 
Periodontal infection contributes to atherosclerosis and thromboembolic events by repeatedly challenging the vascular endothelium and arterial wall with bacterial LPSs and proinflammatory cytokines.

Gram-negative bacteria with their associated LPSs cause infiltration of inflammatory cells into the arterial wall along with proliferation of arterial smooth muscle and coagulation intravascularly ${ }^{6}$. Naturally occurring atheromatosis have similar identical changes. There is strong evidence that periodontal bacteria disseminate from the oral cavity to the systemic vasculature, can be found within distant tissues and can live within those affected tissues. Dissemination of periodontal bacteria can induce atherosclerosis in distant vessels. Increased risks for thickening of the walls of coronary arteries have been seen in patients with periodontitis. In several studies of atheromas obtained from humans during endarterectomy, more than half of the lesions contained periodontal pathogens and many atheromas contained multiple different periodontal species ${ }^{6}$. Periodontal diseases result in chronic systemic exposure to products of these organisms. Low-level bacteremia may initiate host responses that alter coaguability, endothelial and vessel wall integrity and platelet function, thereby resulting in atherogenic changes and possible thromboembolic events under the arterial intima ${ }^{4}$.

Some individuals with high proportions of pathogenic organisms and heavy plaque accumulation appear relatively resistant to bone and attachment loss. Others having a hyperinflammatory monocyte and macrophage phenotype (MØ+) develop extensive periodontal destruction in the presence of small amounts of plaque and low proportions of putative pathogens ${ }^{6}$. These hyperinflammatory monocytes and macrophages in response to bacterial LPSs secretes significantly increased levels of proinflammatory mediators e.g., TNF- $\alpha$, PGE2, IL-1 compared to patients with a normal monocyte and macrophage phenotype. Patients with aggressive periodontitis, refractory periodontitis and type 1 diabetes mellitus often possess the МØ+ phenotype, which appears to be under both genetic and environmental control. The monocyte and macrophage cell line is intimately involved in the pathogenesis of both periodontal disease and atherosclerosis. Diet-induced elevations in serum lowdensity lipoprotein levels up-regulate monocyte and macrophage responsiveness to bacterial LPSs. Thus, elevated lowdensity lipoprotein levels, which are a known risk factor for atherosclerosis and CHD, may increase the secretion of destructive and inflammatory cytokines by monocytes and macrophages. This may result not only in the propagation of atheromatous lesions, but also in enhanced periodontal destruction in the presence of pathogenic organisms. The presence of an $\mathrm{M} \emptyset+$ phenotype may place patients at risk for both CHD and periodontitis ${ }^{4}$. In response to inflammatory or infection liver produces acute-phase proteins such as $\mathrm{C}$ reactive protein and fibrinogen which act as inflammatory markers. These inflammatory markers induce monocytes and macrophages to produce tissue factor, which increases blood coaguability by stimulating the coagulation pathway. Also it's seen that CRP stimulates the complement cascade, further increasing inflammation. Both elevations of both serum CRP and fibrinogen levels act as risk factors for cardiovascular disease and are elevated in patients with periodontitis as compared with patients without periodontitis ${ }^{4}$. Thus, as seen periodontal diseases have both indirect effects that stimulate changes in the cardiovascular system e.g., elevation of systemic inflammatory responses and direct effects on the major blood vessels e.g., atheroma formation. Serum levels of inflammatory mediators and markers are assessed before and after treatment to decrease periodontal inflammation and it's seen that serum levels of IL-6 and CRP are reduced after scaling and root planing. Also periodontal treatment leads to reduction in 
serum levels of CRP, IL-6, fibrinogen and TNF- $\alpha$. Inflammatory periodontal disease (compared with periodontal health) is also seems to be linked with altered vascular endothelial function which is major risk factor for acute thrombotic events. After scaling and root planing with a resultant decrease in periodontal inflammation, normal functioning of vascular endothelial function occurs and also markers of vascular health improve significantly over time. These results suggest that periodontal inflammation adversely affects the health of the vascular endothelium, whereas a reduction in inflammation improves endothelial health ${ }^{4}$. Lowering of dietary lipids results in decreased levels of plaque. However, the effect of diet-induced lipid lowering on plaque accumulation is blunted by $\mathrm{P}$. gingivalis oral infection by altering the intestinal microbiota associated with atherosclerosis development. The dysbiosis induced by dietary lipids and $\mathrm{P}$. gingivalis oral infection is characterized by a decreased community complexity and overgrowth of specific commensal bacteria.

\section{Association Between Periodontal Inflammation And Cardiovascular Diseases}

Patients with periodontal disease share many of the same risk factors as patients with cardiovascular disease including diabetes, smoking, age, gender (predominantly male), lower socioeconomic status, stress and smoking. As tobacco smoking and high levels of LDL or "bad" cholesterol have long been associated with cardiovascular disease and increased levels of CRP is important risk factors for cardiovascular diseases, increasing its risk two fold to five fold, similarly periodontal disease is associated with cardiovascular risk factors including CRP and plasma fibrinogen. Individuals with periodontitis have increased systemic levels of CRP and fibrinogen evan after adjustment of other factors like education, sex, age, body mass index, dental calculus, ethnicity, family size, family history of myocardial infarction, diabetes, tobacco and alcohol use.

Individuals with low attachment loss and low tooth loss exhibited increased prevalence of coronary heart disease in comparison to individuals with both high attachment loss and high tooth loss. Thus, clinical signs of periodontitis are linked with increased chances of having subclinical atherosclerosis, coronary heart disease and further exposures to specific periodontal pathogens which increases the risk for atherosclerosis in non-smoking and smoking individuals.

It was found that mean carotid plaque thickness was significantly greater among dentate subjects with severe periodontal bone loss as compared to those with less bone loss $(<50 \%)$. The investigators found that cumulative periodontal bacterial burden was significantly related to carotid intima-media wall thickness after adjusting for cardiovascular disease risk factors. Individuals with greater than the age specific median number of teeth lost exhibited a significantly increased risk of death from myocardial infarction and stroke.

\section{Mechanisms for the Association Between Periodontitis and Cardiovascular Diseases}

The following describes three popular proposed mechanisms: infection, molecular mimicry and systemic inflammation.

\section{1) Infection}

Oral and periodontal bacteria can easily gain access to the circulation and colonize distant sites. P. gingivalis has cell wall components such as endotoxins and adhere to and invade several cell types including endothelial cells ${ }^{7}$. In addition to P. gingivalis, T. forsythus and A. actinomycetemcomitans have also been isolated from atheromas. Several viruses including cytomegalovirus and hepatitis A virus and bacteria like Helicobacter pylori, Chlamydophila pneumoniae have also been found in atheromas and their presence has 
been proposed as a precipitating factor in ASVD events. Unstable angina combined with myocardial infarction reported no benefit of long-term antibiotic therapy as periodontopathic bacteria exist in a biofilm within the periodontal pocket and not within the host tissues making systemic antibiotics not totally effective in decreasing the oral load of these bacterial species within an individual ${ }^{7}$.

\section{2) Molecular Mimicry}

Molecular mimicry or immune cross-reactivity occurs when an immune response to antigenic structures (epitopes) present on a pathogenic species cross-react with host cell epitopes. Both the pathogen and host cells that display the cross-reacting epitope are targeted by the immune response. Molecular mimicry occurs since the strategies to remove $\mathrm{T}$ and $\mathrm{B}$-cells that are self-reactive during immune cell development are imprecise. For example, patients suffering ASVD events had a greater total burden of $\mathrm{P}$. gingivalis, A. actinomycetemcomitans and $\mathrm{T}$. forsythia. One of several proteins expressed by host cells when injured or under conditions of metabolic stress is human heat shock protein 60. Usually under conditions of endothelial dysfunction, endothelial cells express heat shock protein. Human heat shock protein 60 shares epitopes with GroEL, a protein expressed by $\mathrm{P}$. gingivalis and other periodontopathic bacteria. Therefore an adaptive immune response generated to bacterial GroEL could also cross-react with human heat shock protein 60 . The crossreactive immune response could result in continued injury to the intima, facilitating endothelial dysfunction and atherosclerotic progression ${ }^{7}$.

\section{3) Inflammation}

The moderate to severe periodontitis leads to generation of the pro-inflammatory cytokines TNF- $\alpha$, IL-1 and IL-6 whose elevated systemic levels in turn induce an acute phase response. Moderate to severe periodontitis leads to elevated CRP, fibrinogen, blood glucose, white blood cell counts, total cholesterol, triglycerides and LDL and depressed HDL which are components of the acute phase response, are recognized as ASVD risk factors ${ }^{7}$.

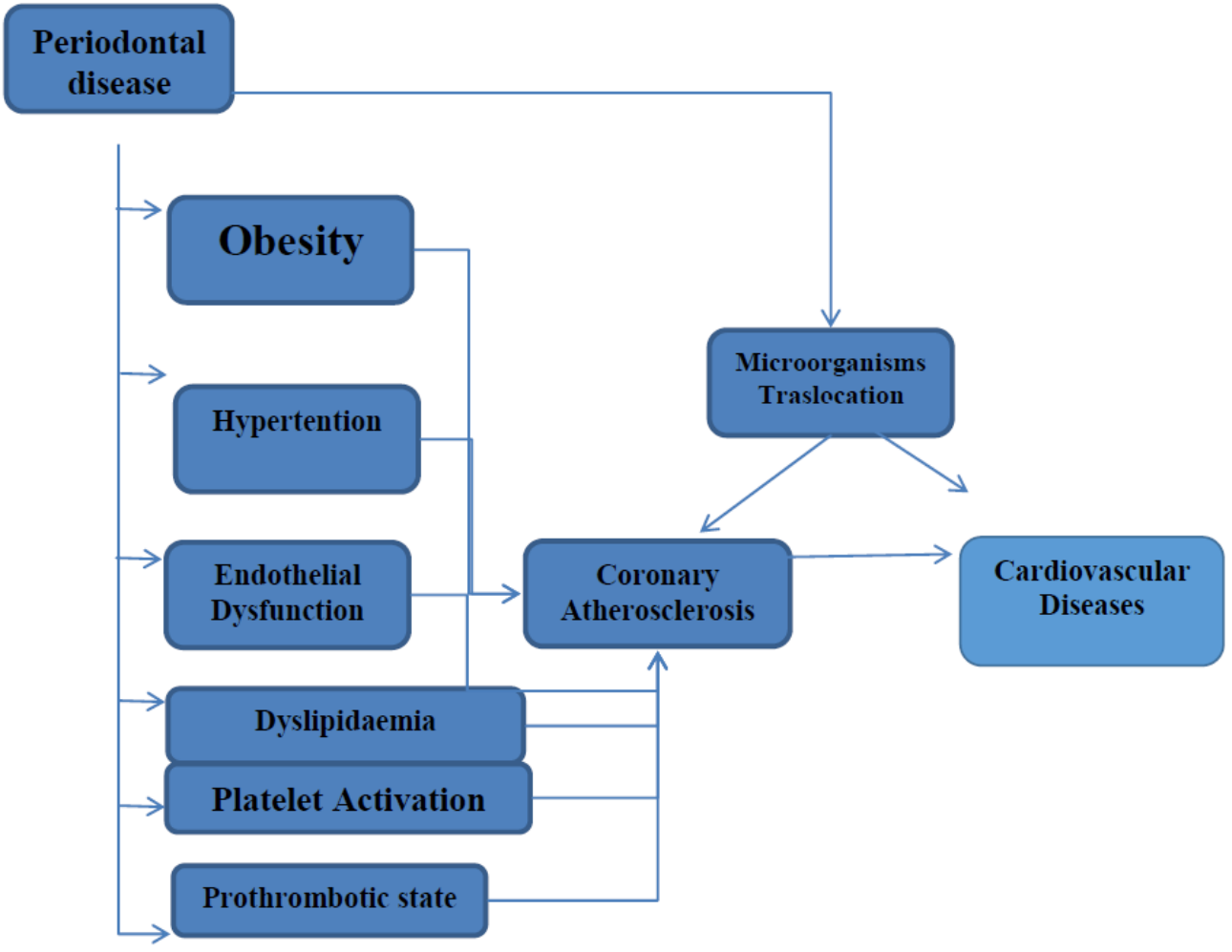

Fig 2: Mechanisms for the Association Between Periodontitis and Cardiovascular Diseases 


\section{Are severe and moderate periodontitis associated with acute myocardial infarction ?}

Periodontitis can predispose to myocardial infarction as a result of a number of possible biological mechanisms that is responsible for the increased inflammatory response in atheromatous lesions. Various mechanisms include high hemostatic and thrombotic markers that promote a prothrombotic and inflammation status; dyslipidemia promotion with a consequent increase in proinflammatory lipid classes and subclasses; increased systemic levels of inflammatory mediators stimulated by bacteria or their products in distant sites of the oral cavity, cross-reactive systemic antibodies that promote inflammation and interact with the atheroma; and common genetic factors present in both diseases leading to increased inflammatory responses ${ }^{8}$. Studies have proved association between moderate and severe periodontitis severity levels and AMI, after adjustment of following confounders: schooling level, current smoking habit, hypercholesterolemia, and BMI. After adjustments for relevant confounders, this association did, however, remain more among females ${ }^{9}$, and thus underlining the importance of considering poor dental health when evaluating cardiovascular risk, especially in younger females, the pronounced association among younger females deserves attention. In patients with moderate and severe periodontitis, the chance of having AMI was approximately two to four times higher than among those without periodontitis ${ }^{8}$. However, the chance of having AMI was higher among those individuals with severe periodontitis than with moderate periodontitis demonstrating a possible relationship among the levels of periodontitis severity on AMI.

The results that more severe periodontitis has a higher strength of association than moderate periodontitis in relation to myocardial infarction lead to argue for provision of dental care for individuals with more severe levels of periodontitis, as these conditions are more related to myocardial infarction ${ }^{8}$.

\section{Effect of Periodontal therapy on Cardiovascular events}

Periodontitis patients with favorable clinical responses to non-surgical periodontal therapy (i.e. scaling and root planing, topical and oral antimicrobials plus mechanical retreatment) exhibited concomitant improvements in flowmediated dilatation of the brachial artery and serum C-reactive protein concentrations. Surrogate cardiovascular disease outcomes like serum biomarkers and endothelial dysfunction can be improved by Periodontal therapies.

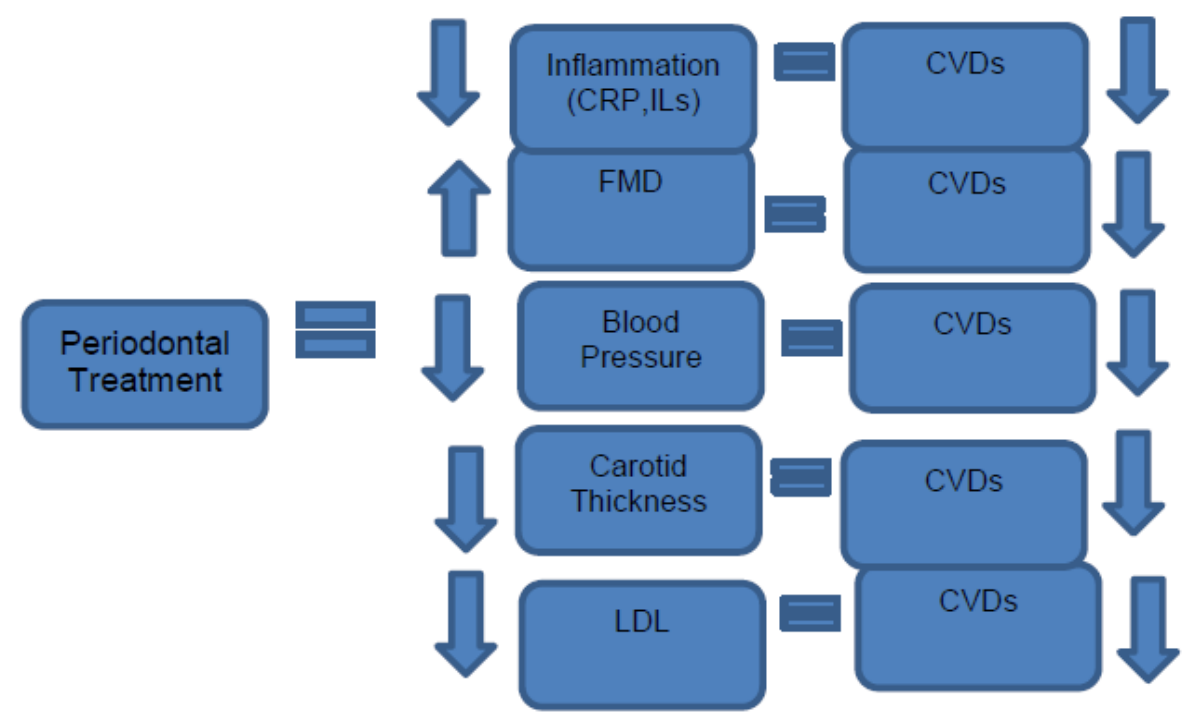

Fig 3: Impact of Periodontal treatment on cardiovascular diseases 
Management of periodontitis is sufficient to decrease platelet hyperreactivity and reduction of $\mathrm{P}$-selectin and CD63 plasma values, as well as decrease of platelet-leukocyte complexes formation in response to Porphyromonas gingivalis stimulation.

Furthermore, treatment of periodontal patients is associated with a reduction of CRP levels 1 year after therapy. Anti-infective periodontal therapy, such as scaling and root planing and administration of subgingival doxycycline as an adjunct to scaling and root planing, results in a reduction in levels of CRP and fibrinogen to normal levels. Periodontal maintenance therapy aimed at reducing levels of subgingival $P$. gingivalis maintains the levels of CRP and fibrinogen within normal limits and also mechanical periodontal therapy along with subgingival administration of minocycline decreased levels of CRP and TNF- $\alpha$ in patients with increased risk for atherosclerosis. Results from these studies have quite significant implications for management of periodontal infections in patients with periodontal disease and increased risk for cardiovascular disease.

\section{Influence of Cardiovascular Disease on Periodontal Condition}

Three main areas can be identified by the periodontist, when treating a patient with Cardiovascular disease are as follows:

1. the effect of medication on either the periodontium or the delivery of periodontal care;

2. the risk of Infective Endocarditis (IE) arising from dental and periodontal procedure

3. the possibility that the patient's periodontal condition may have contributed to the cardiovascular problem.

Calcium-channel blockers and badrenoceptor blockers cause gingival overgrowth and tooth demineralisation respectively. Stoppage of anticoagulant therapy before periodontal procedures can put patients at a greater risk of thromboembolic disorders than the risk of prolonged bleeding. The association between dental treatment and infective endocarditis remains a controversial issue as it has been seen that bacteraemia due to patient's oral hygiene practices is more likely can cause endocarditis than any periodontal treatment or procedure ${ }^{10}$.

\section{Drug Therapy}

In a few areas patient's systemic medication can affect either the periodontium or the delivery of periodontal care. Examples are as follows: drug-induced gingival overgrowth caused by calciumchannel blockers; the effect of badrenoceptor blocking and diuretic drugs on calculus formation; managing patients on anticoagulant and antiplatelet drugs.

\section{A) Calcium-channel blocker-induced gingival overgrowth}

Virtually all the calcium-channel blockers have been cited as causing gingival overgrowth. The prevalence varies from 3\% to as high as $43 \%$ (Nery et al 1995, Jorgensen 1997). Development of gingival changes is three times more likely in male than females patients. An unusual finding with gingival overgrowth induced by calcium-channel blockers is the drug sequestration in gingival crevicular fluid (GCF). The increase extent of gingival inflammation is due to increased concentration of nifedipine and amlodipine in GCF. Patients taking nifedipine had significant overgrowth, whilst the prevalence of gingival changes in patients medicated with either amlodipine or diltiazem was slightly more or no greater than a non medicated control group.

\section{B) b-Adrenoceptor blockers, diuretics and calculus formation}

Systemic medication with either a bblocker or a diuretic has an inhibitory effect on calculus formation or re-formation after a prophylaxis (Turesky et al 1992, Breuer et al 1996). Presence of the drug in saliva 
increases the rate of crystallisation by a physicochemical mechanism, or by the altering the composition of saliva and thus affects calculus formation directly and indirectly ${ }^{10}$.

\section{C) Anticoagulants and antiplatelet drugs}

To prevent thromboembolic disorders both anticoagulants and antiplatelet drugs including warfarin, lowdose aspirin and dipyridamole are used in patients with cardiovascular diseases. From the periodontal perspective, patients on these drugs pose a problem of an increased risk of haemorrhage arising from any procedure. Bringing the INR down to below 2.5 by consulting the physician and arranging for cessation of their warfarin therapy for 48 hour prior to any elective procedure is to be carried out to safe-gourd before any surgical procedure ${ }^{10}$.

\section{CONCLUSION}

The review article shows that there is a significant association between CVD and periodontitis. While there is still a lack of sufficient evidence for the causal relationship between CVD and periodontitis, it is undeniable that this association is of great importance because of the beneficial effect on reduction of the risk of CVD by preventing or treating periodontal disease.

Patients with Cardiovascular Diseases have poor periodontal status than patients without coronary heart disease. In same way bleeding on probing, severe periodontitis and more plaque accumulation are associated with Cardiovascular Diseases. Periodontitis is a risk factor and negatively affects the degree of postinfarction left ventricular damage proving that there is an inflammatory link between these two diseases. Periodontal disease may unfavorably modulate the cardiovascular risk, whereby patients with periodontitis may also be characterized by aprothrombotic state which is characterised by increased frequency of overweight, hypertension, endothelial dysfunction, dyslipidaemia and platelet hyper-reactivity. Additionally translocation of periodontal microorganisms into the bloodstream, and their accumulation with in atherosclerotic plaques would further contribute to enhance plaque instability and the chances of developing acute ischemic coronary events. Interesting evidence is also emerging that local or systemic statins administration, which is a known anti-inflammatory agent, and modify the severity of the systemic inflammation, could be beneficial for safeguarding periodontal health, thus enlightening the intriguing relationship existing between CVD and periodontitis ${ }^{11}$.

Almost all of the studies provided evidence of an association between CVD and periodontitis and especially worsened periodontal status in CVD patients as CVD patients had a higher chances of having and developing periodontitis than did the control subjects. Further studies are therefore necessary to illustrate the physicopatholological mechanisms and take the confounding factors into account so as to allow better planning of prevention programme.

Thus it seems hence reasonable to conclude that management of periodontal disease generates favourable impacts on cardiovascular risk factors, thus ultimately lowering the risk of developing CVD. Thus concluding that Treat Gums, benefit Heart.

\section{Acknowledgement: None}

Conflict of Interest: The author(s) declared no potential conflicts of interest with the research, authorship, and/or publication of this article.

Source of Funding: The author(s) received no financial support for the research, authorship, and/or publication of this article.

\section{REFERENCES}

1. Reynolds HR, Craig RG. Atherosclerotic Vascular Disease and Periodontal Disease. A Clinician's Guide to Systemic Effects of Periodontal Diseases 2016;39-51. 
2. Shi Q, Zhang B, Huo N, et al. Association between Myocardial Infarction and Periodontitis: A Meta-Analysis of CaseControl Studies. Frontiers in Physiology. 2016;7:519.

3. Hajishengallis G, Chavakis T. Local and systemic mechanisms linking periodontal disease and inflammatory comorbidities. Nat Rev Immunol. 2021; 21:426-440.

4. Kovacic S, Bakran M. Genetic susceptibility to atherosclerosis. Stroke Res Treat. 2012; 2012:362941.

5. Mealey BL, Klokkevold PR. Impact of Periodontal Infection on Systemic Health. Clinical Periodontology2007;10:315-320.

6. Newman MG, Takei HH, Klokkevold PR, Carranza FA. Impact of Periodontal Infection on Systemic Health. Carranza's Clinical Periodontology2006;10:219-224

7. Ryan ME, Raja VS, Sussman SK, Reynolds HR, Craig RG. A Clinician's Guide to Systemic Effects of Periodontal Diseases 2016;1:19-37.
8. Gomes-Filho IS, Coelho JMF, Miranda SS, et al. Severe and moderate periodontitis are associated with acute myocardial infarction. J Periodontol. 2020;1-9.

9. Nordendah E, Gustafsson A, Norhammar A et al. Severe Periodontitis Is Associated with Myocardial Infarction in Females. JDR2018;1-8.

10. Seymour RA, Preshaw PM, Thomason JM, Ellis JS, Steele JG. Cardiovascular diseases and periodontology. J Clin Periodontol 2003; 30: 279-292.

11. Nocini R, Favaloro EJ, Sanchis-Gomar F, Lippi G. Periodontitis, coronary heart disease and myocardial infarction: treat one, benefit all. Blood Coagul Fibrinolysis. 2020 Sep;31(6):339-345.

How to cite this article: Nirola A, Batra P, Singla $\mathrm{P}$ et.al. Is periodontitis a risk predictor with adverse outcomes for cardiovascular diseases: treat gums, save heart. Int J Health Sci Res. 2021; 11(9): 237-245. DOI: https://doi. org/10.52403/ijhsr.20210936 